\title{
Physical Intimate Partner Violence Among Pregnant Women in Ofla District, Tigray, Ethiopia
}

\author{
Girmay Adhena ${ }^{1,{ }^{*}}$, Lemessa Oljira ${ }^{2}$, Tesfay Temesgen $^{3}$ \\ ${ }^{1}$ Department of Reproductive Health, Tigray Regional Health Bureau, Tigray, Ethiopia \\ ${ }^{2}$ School of Public Health, College of Health and Medical Science, Haramaya University, Harar, Ethiopia \\ ${ }^{3}$ Department of Midwifery, College of Health Science, Dilla University, Dilla, Ethiopia \\ Email address: \\ girmayrh@gmail.com (G. Adhena) \\ ${ }^{*}$ Corresponding author
}

To cite this article:

Girmay Adhena, Lemessa Oljira, Tesfay Temesgen. Physical Intimate Partner Violence Among Pregnant Women in Ofla District, Tigray, Ethiopia. American Journal of Health Research. Vol. 8, No. 4, 2020, pp. 69-77. doi: 10.11648/j.ajhr.20200804.13

Received: June 1, 2020; Accepted: June 18, 2020; Published: August 4, 2020

\begin{abstract}
Background: Physical violence by an intimate partner during pregnancy is the most common, hidden, and serious public health problem worldwide particularly in Africa. However, this issue has received little attention in Ethiopia. This study assessed the magnitude of intimate partner physical violence and associated factors among pregnant women in Ofla District, Tigray, Ethiopia. Methods: Institutional based cross-sectional study was conducted among pregnant women who attended antenatal care in public health facilities. Participants were selected using a systematic random sampling technique. Pretested, structured, and face to face data collection process was done using a standardized World Health Organization multi-country questionnaire. Bivariable and multivariable logistic regression analyses were carried out. Odds ratio with $95 \%$ to show the strength and finally variables with $\mathrm{p}$-value $<0.05$ was considered as statistically significant. Results: The overall proportion of physical violence by an intimate partner during the current pregnancy was $13.4 \%$. Violence was associated with being unmarried [(AOR $=3.23,95 \% \mathrm{CI}:(1.19,8.82)]$, being rural dweller [( $\mathrm{AOR}=3.99,95 \% \mathrm{CI}$ : $(1.44,11.1)]$, undesired pregnancy by intimate partner [(AOR=4.18, 95\% CI: $(1.7,10.29)$ ], having alcoholic partner [(AOR=3.3, 95\% CI: $(1.51,7.19)]$, spouse's multiple sexual partners status $[(\mathrm{AOR}=3.77,95 \% \mathrm{CI}:(1.6,8.87)]$. Conclusions: Considerable proportion of pregnant women experienced physical violence by their intimate partner during a recent pregnancy. Addressing male involvement in sexual and reproductive health issues and empowering women in the community through awareness and intervention measures are very important to minimize the problem.
\end{abstract}

Keywords: Physical Violence, Intimate Partner, Pregnant Women, Ethiopia

\section{Background}

Intimate partner physical violence is any non-accidental act or behavior threatened or actual against a woman or man within a relationship causing in or has a high likelihood of resulting in injury, trauma, physical suffering, and death [1]. It is the most common form of violence against women and major public health issues globally [2]. It is also a complex and silent pandemic that has detrimental effects on female victims' mental health and causes a significantly higher use and cost of health care services [3]. Physical violence during pregnancy is the most devastating problem for both mother and fetus because of its morbidity and mortality $[2,4]$.
More than one-third of women have experienced physical and/or sexual violence worldwide and out of this number, most of these women have experienced by their intimate partner in their lifetime [5]. Women experienced lifetime physical intimate partner violence ranges from $6 \%$ to $48 \%$ globally $[6,7]$. In low- and middle-income countries ranges between $13 \%$ and $68 \%$ of women have experienced at least one incident of physical violence by their intimate partner [8] It is also highest in sub-Saharan Africa, 38 to $83 \%$ of women were abused by their intimate partners [2].

The prevalence of physical intimate partner violence in pregnancy ranges between $1 \%$ in Japan to $28 \%$ in Peru Province, with the majority of sites ranging between $4 \%$ and $12 \%$ [8]. Demographic and Health Surveys and the 
International Violence against Women Survey found that prevalence rates for intimate partner physical violence during pregnancy between $2 \%$ in Australia, Denmark, Cambodia, and Philippines to $13.5 \%$ in Uganda, with the majority ranging between $4 \%$ and $9 \%$ [9]. Other studies also showed that the highest prevalence in Egypt with $32 \%$, followed by India (28\%), Saudi Arabia (21\%), and Mexico (11\%) [6]. A review of clinical studies from Africa reports the prevalence rates of $23-40 \%$ physical intimate partner violence during pregnancy [10].

Although Ethiopia has ratified many international and continental agreements that promote and protect women's rights and has established specific legal measures and actions to address violence against women, this issue continues to be a major challenge and a threat to women's empowerment. About twenty-three percent of reproductive age women have experienced physical violence since age 15 years old, and $15 \%$ have experienced physical violence within a year. Out of this, $68 \%$ report their current partners as perpetrators of physical violence [11]. Intimate partner violence (IPV) is common in Ethiopia both in urban and rural families. About $68-81 \%$ of women agree on wife-beating if a husband has justified in at least one of the specified situations in Ethiopia [12].

Physical violence by an intimate partner during pregnancy is associated with fatal and non-fatal adverse health outcomes for the pregnant woman and her baby due to the direct trauma of abuse to a pregnant woman's body, as well as the physiological effects of stress from current or past abuse on fetal growth and development [13]. There is also a particular concern if the abdomen is targeted during pregnancy due to the risk of injury to the fetus [12]. Women who have been physically abused by their partners report higher rates of health problems [1]. Apart from the violations of human rights, domestic violence is associated with various poor health outcomes for the newborn such as low birth weight, premature birth, fetal death, placental damage, fetal trauma, preterm labor and among the women and girls include suicide, homicide, mental illness, physical injuries, disability. Moreover, violence also associated with various poor reproductive health conditions such as induced abortion, abdominal pain, bleeding, human immune virus (HIV), and other sexually transmitted infections [1, 8, 13-15].

Although the prevalence of intimate partner physical violence (IPPV) varies across countries, the factors associated with an increased risk of IPPV are similar. These may include the desire of pregnancy, attitudes towards IPV, educational status, plan of pregnancy, discussion making power, socio-economic status, alcohol consumption by the partner, and behavioral factors [14-16].

Even though the consequences of physical violence during pregnancy are worst, studies conducted so far were mostly focused on IPV of non-pregnant women. The magnitude of IPPV during pregnancy and its associated factors were not well addressed in sub-Saharan countries including Ethiopia, particularly in the study area. Therefore, this study was designed to assess the prevalence of IPPV and associated factors among pregnant women in Ofla District, Tigray,
Ethiopia.

\section{Methods}

\subsection{Study Area and Period}

The study was conducted in Ofla District which is one of the Districts in Tigray Regional State, Ethiopia. It is located $619 \mathrm{~km}$ away from Adis Ababa, the capital city of Ethiopia. Ofla District is bordered to Machew by the North, Raya Azebo by the East, Amhara Regional State by the West, and Alamata by the south. The District is the second rank in the size of the population in the Region. The total population of the District was 175,659 . Of this, 87,221 (49.65\%) were males and $88,438(50.35 \%)$ were females. Regarding the health infrastructure, there was one general hospital, seven health centers, and 25 health posts that serve the population in the District [17]. The study was conducted from March 1$30 / 2019$.

\subsection{Study Design and Population}

An institutional-based cross-sectional study was conducted. All pregnants who were following ANC in the public health facilities of Ofla District were the source population. Pregnant women who were attending ANC in the public health facilities during the data collection period constituted the study population. However, pregnant women with severe major danger signs of pregnancy during the data collection period were excluded.

\subsection{Sample Size Calculations and Sampling Procedures}

The sample size was calculated for the first objective (magnitude) and the second objective (factors). For the first objective using single population formula $\left(n=Z^{2} p(1-p) / d^{2}\right)$, where $\mathrm{z}$ is the normal standard deviation set at 1.96 , with a confidence level specified at $95 \%$ and a tolerable margin of error (d) at 5\%, considering $10 \%$ nonresponse rate and prevalence of IPPV (p) 20.6\% from a previous similar study conducted in Shire EndaSelassie, Tigray, Ethiopia [16]. The calculated sample size for this was 251 and by adding the non-response rate finally it gives $276(251+251 * 10 \%)$. The sample size was also calculated for the second objective by using the Epi-info version-7 with the assumption of a $95 \%$ confidence interval, $80 \%$ power, $5 \%$ degree of precision, and $10 \%$ non-response rate. Since the sample size of the second objective (543) was higher than the first objective (276), the total sample size finally used in this study was 543 .

For the sampling procedure, all public health facilities (seven health centers and one general hospital) in Ofla District were considered. Pregnant women who attended ANC clinics for an average of six months before the study period were used to estimate recruitment from each health facility. The average client flow per month and day in the public health facilities of Ofla District were considered and the population proportion sample size allocation was done to each health facility. A systematic random sampling (SRS) technique was used to select study participants and every 
third $\left(\mathrm{K}^{\mathrm{th}}=3\right)$ pregnant woman was chosen based on their visiting order until the sample size was met.

\subsection{Data Collection Tool and Procedures}

To ensure data quality a standardized, structured WHO Multicountry study questionnaire for assessing women's health and violence was used for data collection [8]. The questionnaire was translated into the local language (Tigrigna) to establish accuracy, cognitive understanding, and cultural acceptability and pretested on 5\% [27] of the total sample size was adapted and used. The respondents were asked questions about their experience of specific acts of physical violence by their current intimate partner. The questionnaire has five items of physical violence to measure the outcome variable (IPPV) and the response to each item was either "Yes" or "No". Current prevalence is the proportion of pregnant women reporting at least one act of physical violence during the current pregnancy was considered as incident cases of IPPV victimization. The questionnaire also covered socio-demographic, behavioral, discussion making capacity, and attitudinal characteristics of women and their current intimate partners. Data were collected by 10 midwives who were all females which creates an opportunity for disclosure of violence by the women. Two public health officers and the principal investigator supervised the data collection procedures. Data collectors and supervisors were trained for two days on interviewing techniques, the purpose of the study, the importance of privacy, the sensitivity of the issue, discipline and approach to the interviewees, and confidentiality of the respondents. The collected data were cross-checked on each day of activity for consistency and completeness, double-entry of data and cleaning of data using frequency, sorting, and listing to identify any missed value and outlier were made, and identified errors were cross-checked with the original questionnaire. One data clerk was used for data support.

\subsection{Operational Definitions}

Intimate partner physical violence (IPPV): Woman's report at least one threat or act of slapped, pushed, shoved, pulled, throw something that could hurt, choked, burning on purpose, hit abdomen with a fist or with something else and if a gun, knife or any other weapon was used against a woman by an intimate partner during current pregnancy [8].

Intimate partner: Current husband, co-habited, or boyfriend [18].

Decision-Making Power: High decision-making power is when a woman made a sole discussion alone or with here intimate partner on " how to use the money earned by her or her partner on healthcare for herself, major household purchases, and visits to her family or relatives. Otherwise (partner alone or other person decides in those issues) it categorized as low discussion maker [19].

Acceptance of IPV: Women were asked about their attitudes towards IPPV when did they accept violence committed by an intimate partner (argues with him, burns the food, goes without telling him, failing to complete housework and refusal of men's request for sex). Acceptance of at least one issue is considered as the women accept violence otherwise the women do not accept [20].

\subsection{Data Processing and Analysis}

Data coding, entry, and cleaning were processed using Epidata version 4.1 statistical software and the analysis was made using SPSS version 20. Descriptive statistical analyses such as frequencies, measures of central tendency, and measure of variability were used to describe the characteristics of participants and their intimate partners. Then information was presented using summary measures, narratives, tables, and figures. The principal component analysis was used to produce Wealth Quintiles to show and reveal the socio-economic status of the households. The bivariable analysis was carried out to see the association of each independent variable with the IPPV. Variables with pvalue $<0.25$ in the bivariable analysis were taken into the multivariable analysis model to control all possible confounders. Co-linearity test was carried out to see the correlation between independent variables using the standard error. A Hosmer-Lemeshow and Omnibus tests were conducted to test model goodness of fit. The multivariable analysis was performed in the binary logistic regression up on controlling for the possible confounding factors. Odds ratios with $95 \%$ CI were reported to show the strength and direction of the associations. Finally, variables with a p-value less than $0.05 \quad(\mathrm{p}<0.05)$ in the logistic regression were considered as statistically significant.

\subsection{Ethical Considerations}

The study followed the WHO guidelines for researching violence against women and girls. Ethical clearance was secured from Haramaya University College of Health and Medical Sciences Institutional Health Research Ethics Review Committee (IHRERC). A letter of permission was obtained from the Ofla district health office. Informed, written, voluntary, and signed consent was obtained from each head of the health facilities and each participant after clearly informing them about the purpose, risk, and benefit of the study. The interview was conducted in a separate and calm room to ensure the safety and confidentiality of the study participants.

\section{Result}

\subsection{Background and Other Characteristics of Women and Their Intimate Partners}

In general, a total of 538 out of 543 pregnant women have completed the interview about their experiences of violence making a response rate of $99.08 \%$. The mean age of participants was $27.4( \pm 6.2 \mathrm{SD})$ years. About half $(48.1 \%)$ of them were between the age of 25 and 34 years old. Nearly nine out of ten $(88.5 \%)$ pregnant women were married. The majority $(63.2 \%)$ of participants were rural dwellers, about 
503 (93.5\%) were Orthodox in their religion and $531(98.7 \%)$ were Tigray in their ethnicity. More than half of the pregnant women $(50.7 \%)$ had no formal education, about one third (33.4\%) of the respondents' wealth quintile was lived into households with medium and nearly half $(48.9 \%)$ were housewives in their occupational status. About $86(16 \%)$ women reported that the current pregnancy was unplanned and one third $(33.5 \%)$ of participants had low discussionmaking capacity in the household issues. The majority (66.9\%) of participants have a positive attitude (acceptance) towards any form of violence committed by an intimate partner by justifying reasons for men's violent act. Regarding intimate partners of pregnant women's characteristics, about near to half (45.4\%) were illiterate in their educational status, $28(5.2 \%)$ were smokers and $58(10.8 \%)$ had another sexual partner. More than half $(54.1 \%)$ of partners of pregnant women were alcohol users and Seventy three (13.6\%) of pregnant women reported that their intimate partner did not desire the current pregnancy (Table 1).

Table 1. Background and Other characteristics of pregnant women and their intimate partners in Ofla District, Tigray, Ethiopia, 2019 (N=538).

\begin{tabular}{|c|c|c|c|}
\hline Characteristics & Category & Frequency & Percentages \\
\hline \multirow{3}{*}{ Age (years) } & $15-24$ & 193 & 35.9 \\
\hline & $25-34$ & 258 & 48 \\
\hline & $\geq 35$ & 87 & 16.1 \\
\hline \multirow{2}{*}{ Residence } & Rural & 340 & 63.2 \\
\hline & Urban & 198 & 36.8 \\
\hline \multirow{2}{*}{ Marital status } & Married & 476 & 88.5 \\
\hline & Unmarried & 62 & 11.5 \\
\hline \multirow{2}{*}{ Religion } & Orthodox & 503 & 93.5 \\
\hline & Muslim & 35 & 6.5 \\
\hline \multirow{2}{*}{ Ethnicity } & Tigray & 531 & 98.7 \\
\hline & Amhara & 7 & 1.3 \\
\hline \multirow{4}{*}{ Occupational status } & Housewife & 263 & 48.9 \\
\hline & Unemployed & 166 & 30.8 \\
\hline & Merchant & 57 & 10.6 \\
\hline & Government employee & 52 & 9.7 \\
\hline \multirow{3}{*}{ Educational status } & No formal education & 273 & 50.8 \\
\hline & Primary education & 133 & 24.7 \\
\hline & Secondary and above & 132 & 24.5 \\
\hline \multirow{3}{*}{ Educational status of partner } & No formal education & 244 & 45.4 \\
\hline & Primary education & 150 & 27.9 \\
\hline & Secondary and above & 144 & 26.7 \\
\hline \multirow{2}{*}{ Plan of pregnancy } & Planned & 452 & 84 \\
\hline & Unplanned & 86 & 16 \\
\hline \multirow{2}{*}{ Desire for current pregnancy by partner } & Desired & 460 & 85.5 \\
\hline & Undesired & 78 & 14.5 \\
\hline \multirow{2}{*}{ Alcohol use (by partner) } & Yes & 291 & 54.1 \\
\hline & No & 247 & 45.9 \\
\hline \multirow{2}{*}{ Smoking Status (by partner) } & Smokers & 28 & 5.2 \\
\hline & Non-Smokers & 510 & 94.8 \\
\hline \multirow{2}{*}{ Having another wife (by partner) } & Yes & 58 & 10.8 \\
\hline & No & 480 & 89.2 \\
\hline \multirow{2}{*}{ Acceptance of IPV } & Accept & 360 & 66.9 \\
\hline & Do not accept & 178 & 33.1 \\
\hline \multirow{2}{*}{ Decision-making power } & Low & 180 & 33.5 \\
\hline & High & 358 & 66.5 \\
\hline \multirow{3}{*}{ Wealth index } & Low & 179 & 33.3 \\
\hline & Medium & 180 & 33.4 \\
\hline & High & 179 & 33.3 \\
\hline
\end{tabular}

\subsection{The Proportion of Physical Violence}

Out of 538 interviewed pregnant women, 72 [13.4\%; 95\% CI: 10.7\%, 16.5\%] had experienced physical violence by their intimate partner during the recent pregnancy (Figure 1). 


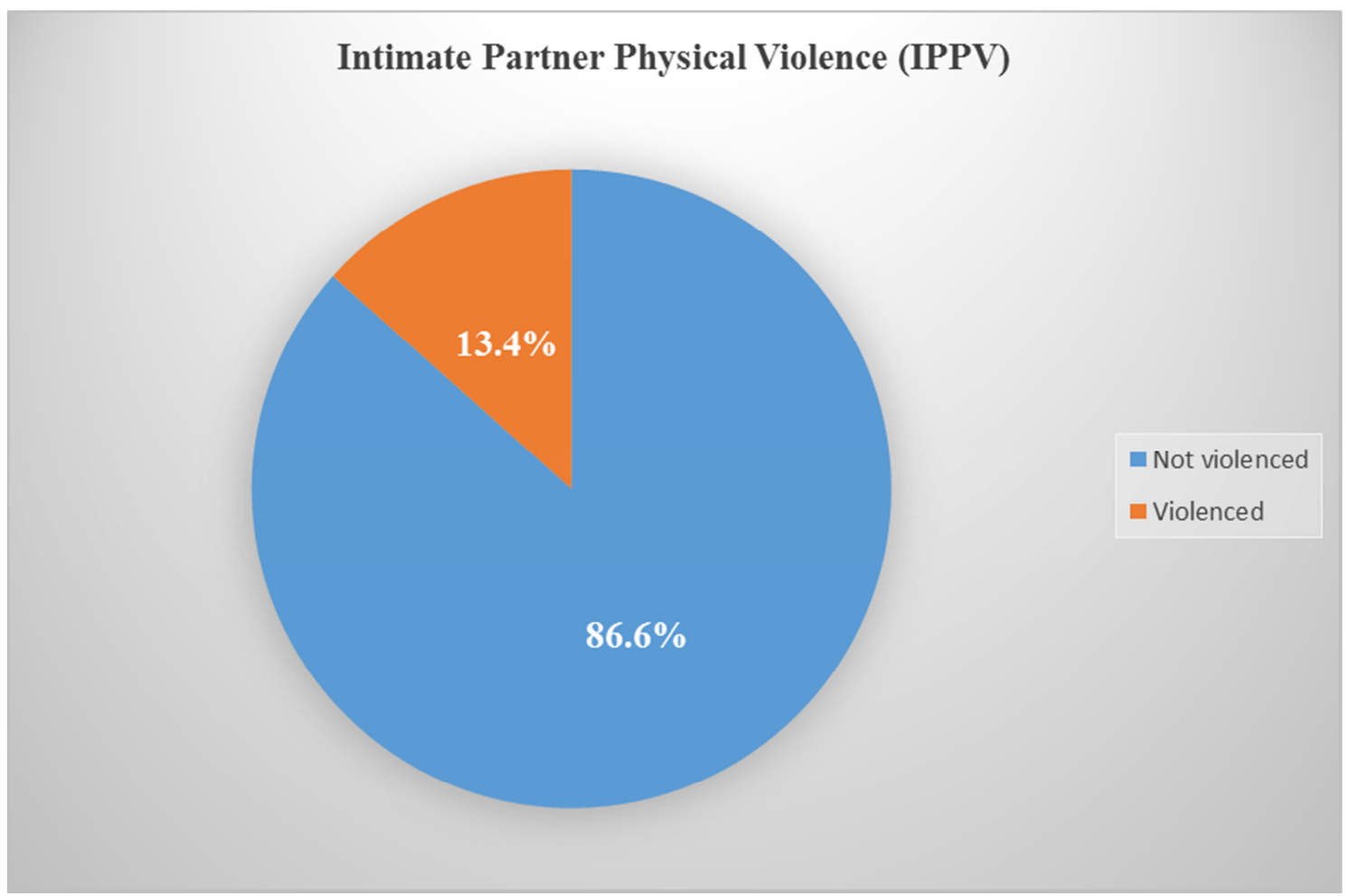

Figure 1. Physical violence by intimate partner among pregnant women in Ofla District, Tigray, Ethiopia, 2019 (N=538).

In this study, the proportion of types of IPPV was assessed. The result showed that Slapped, pushed, or hair-pulling (9.5\%) was the most commonly reported type of physical violence followed by threatened to use or used a gun, knife, or any other weapon against her $(2.2 \%)$ during their recent pregnancy (Table 2$)$.

Table 2. Type of physical violence during pregnancy among pregnant women attending antenatal care services at public health facilities in Ofla District, Tigray, northern Ethiopia, 2019.

\begin{tabular}{lll}
\hline \multirow{2}{*}{ Type of physical violence } & \multicolumn{2}{l}{ Physical violence in the current pregnancy } \\
\cline { 2 - 3 } & Violence & No-violence \\
\cline { 2 - 3 } & Frequency (\%) & Frequency (\%) \\
\hline Slapped, pushed, shoved or pulled & $51(9.5)$ & $487(90.5)$ \\
Threatened to use or used a gun, knife or other weapons & $12(2.2)$ & $526(97.8)$ \\
Choked or burnt on purpose & $10(1.9)$ & $528(98.1)$ \\
Hit abdomen with a fist & $5(0.9)$ & $533(99.1)$ \\
Throw something that hurt the woman & $8(1.5)$ & $530(98.5)$ \\
\hline
\end{tabular}

\subsection{Factors Associated with Physical Violence by Intimate Partner During Pregnancy}

Binary logistic regression showed that age, residence, women's educational status, women's occupation, intimate partner's educational status, intimate partner's alcohol consumption, plan of current pregnancy, marital status, the desire of the current pregnancy by an intimate partner, smoking status of intimate partner, spouse's sexual partners status, acceptance of IPV by women, discussion making power of women and economic status were associated with IPPV in the current pregnancy. In the final model (multivariable analysis), residence, marital status, the desire of pregnancy by a partner, spouse's sexual partner status and intimate partner's alcohol consumption were significantly associated with IPPV during the current pregnancy (Table 3).

\section{Discussion}

The proportion of IPPV during the current pregnancy was (13.4\%; 95\% CI: $10.7 \%, 16.5)$. Being a rural dweller, being unmarried, spouses' having another sexual partner, no interest in the current pregnancy by an intimate partner, and having alcohol drinker partners were significant factors associated with IPPV during their current pregnancy.

This result $(13.4 \%)$ is in line with the findings in Zimbabwe (15.9\%) [10], in Iran (14\%) [21], Hulet Ejunesie (Ethiopia) (11.3\%) [22], Yirgalem (12\%) [14], and Ethiopia Demographic Health Survey (15\%) [11]. However, this result is lower than from the finding in Megacity of South-Asia (35\%) [23], Western Cape (29\%) [24], and findings in Ethiopia such as Shirendasilase $(20.6 \%)$ [16], and in Shimelba $(25.5 \%)$ [25]. The possible reason for the difference might be due to the variation in the accessibility of 
information on gender-based issues and cultures of the study subjects. The other possible reason for the difference might be due to the difference in sample size and sampling method, for example, finding in Western Cape (South Africa) used convenient sampling method and the finding in Shimelba (Ethiopia) also conducted on refugee camps in which the study subjects might be more vulnerable for the victims of violence due to their living environment.

The result in this study is higher than from the finding in Rwanda (10.2\%) [26], and finding in Gonder (Ethiopia) $(8.1 \%)$ [27]. The possible reason for the variation might be due to the difference in study subjects. The study in Gonder (Ethiopia) was on urban dwellers which means that the incidence might decrease due to the accesses of information through media and other sources in urban might decreases the incidence of victims of violence that is living in urban may increase the ability to the know-how on the rules and rights of human being and how to control and combat to those challenges of violence.

This work identified factors associated with physical intimate partner violence during recent pregnancy. Those women who were rural dwellers are 3.8 times more likely to have experience of physical violence by their intimate partner compared to urban dwellers. This is consistent with the findings in Shirendasilase [16], and Gonder [27]. The possible reason might be due to decreased access to information and media in the rural part in which the women decrease their knowhow about violence this might increase the incidence of violence.

The likely hood of physical intimate partner violence among unmarried women was 3.2 times more likely compared to married women in this study. This is supported by the Demographic and Health Survey in Sub Saharan African countries [28]. The possible reason might be due to the communities' perspective in which they give high value for marriage.

Women whose partners did not have an interest in the current pregnancy were about 4.18 times more likely to experience IPPV compared to the pregnant women whose partners desired the current pregnancy. This is consistent with the finding from Huletejuensie (Ethiopia) [22]. The possible reason might be due to no interest in the pregnancy by a partner that may decrease the relationship with pregnant women and mostly they live in an environment with no good relationship. Increasing the need during pregnancy in contrast to different ideas and interests may increase the occurrence of violence.

Table 3. Factors associated with physical intimate partner violence during recent pregnancy in Ofla District, Tigray, Ethiopia, 2019 (N=538).

\begin{tabular}{|c|c|c|c|c|}
\hline \multirow{2}{*}{ Characteristics } & \multicolumn{2}{|c|}{ Violence (IPPV) } & \multirow{2}{*}{ COR 95\% CI } & \multirow{2}{*}{ AOR 95\% } \\
\hline & Violence (\%) & No-Violence (\%) & & \\
\hline \multicolumn{5}{|l|}{ Age } \\
\hline $15-24$ & $17(8.8)$ & $176(91.2)$ & $0.39(0.19,0.82)$ & $0.853(0.225,1.508)$ \\
\hline $25-34$ & $38(14.7)$ & $220(85.3)$ & $0.71(0.378,1.34)$ & $1.066(0.473,2.403)$ \\
\hline $35-49$ & $17(22.1)$ & $70(90.9)$ & 1 & 1 \\
\hline \multicolumn{5}{|l|}{ Residence } \\
\hline Rural & $53(15.6)$ & $287(84.4)$ & $1.74(0.997,3.04)$ & $3.808(1.37,10.58) * *$ \\
\hline Urban & $19(9.6)$ & $179(90.4)$ & 1 & 1 \\
\hline \multicolumn{5}{|l|}{ Employment Status } \\
\hline Unemployed & $32(19.3)$ & $134(80.7)$ & $3.9(1.14,13.32)$ & $1.094(0.126,9.487)$ \\
\hline Housewife & $35(13.3)$ & $228(86.7)$ & $2.52(0.74,8.48)$ & $4.966(0.883,27.94)$ \\
\hline Merchant & $5(8.8)$ & $52(91.2)$ & $0.59(0.095,3.703)$ & $3.22(0.614,16.929)$ \\
\hline Government & $6(11.5)$ & $46(88.5)$ & 1 & 1 \\
\hline \multicolumn{5}{|l|}{ Educational Status woman } \\
\hline No-formal education & 49 (17.9) & $224(82.1)$ & $3.906(1.72,8.88)$ & $2.056(0.446,9.479)$ \\
\hline Primary school & $16(12)$ & $117(88)$ & $2.44(0.97,6.15)$ & $2.95(0.689,12.64)$ \\
\hline Secondary school and above & $7(5.3)$ & $125(94.7)$ & 1 & 1 \\
\hline \multicolumn{5}{|l|}{ Educational Status partner } \\
\hline No-formal education & $46(18.8)$ & $198(81.2)$ & $2.34(1.22,4.5)$ & $1.166(0.424,3.206)$ \\
\hline Primary school & $13(8.7)$ & $137(91.3)$ & $0.96(0.427,2.14)$ & $0.39(0.13,1.192)$ \\
\hline Secondary school and above & $13(9)$ & $131(91)$ & 1 & 1 \\
\hline \multicolumn{5}{|l|}{ Marital Status } \\
\hline Unmarried & $18(29)$ & $44(71)$ & $3.197(1.73,5.93)$ & $3.23(1.19,8.82) *$ \\
\hline Married & $54(11.3)$ & $422(88.7)$ & 1 & 1 \\
\hline \multicolumn{5}{|l|}{ Plan of pregnancy } \\
\hline Unplanned & $36(41.9)$ & $50(58.1)$ & $8.32(4.81,14.38)$ & $2.34(0.926,5.896)$ \\
\hline Planned & $36(8)$ & $416(92)$ & 1 & 1 \\
\hline \multicolumn{5}{|l|}{ Desiredness of pregnancy } \\
\hline Undesired & $37(47.4)$ & $41(52.6)$ & $10.96(6.24,19.23)$ & $4.18(1.696,10.696) * *$ \\
\hline Desired & $35(7.6)$ & $425(92.4)$ & 1 & 1 \\
\hline \multicolumn{5}{|l|}{ Alcohol } \\
\hline User & $62(21.3)$ & $229(78.7)$ & $6.42(3.21,12.82)$ & $3.3(1.514,7.19) * *$ \\
\hline Non-user & $10(4)$ & $237(96)$ & 1 & 1 \\
\hline \multicolumn{5}{|l|}{ Smoking status of partner } \\
\hline Smoker & $14(50)$ & $14(50)$ & $7.79(3.54,17.17)$ & $2.934(0.95,9.066)$ \\
\hline Non-smoker & $58(11.4)$ & $452(88.6)$ & 1 & 1 \\
\hline
\end{tabular}




\begin{tabular}{|c|c|c|c|c|}
\hline \multirow{2}{*}{ Characteristics } & \multicolumn{2}{|c|}{ Violence (IPPV) } & \multirow{2}{*}{ COR 95\% CI } & \multirow{2}{*}{ AOR 95\% } \\
\hline & Violence (\%) & No-Violence (\%) & & \\
\hline \multicolumn{5}{|l|}{ Having another wife } \\
\hline Yes & $28(48.3)$ & $30(51.7)$ & $9.25(5.07,16.87)$ & $3.77(1.604,8.87) * *$ \\
\hline No & $30(6.4)$ & $438(93.6)$ & 1 & 1 \\
\hline \multicolumn{5}{|l|}{ Acceptance of violence } \\
\hline Accept & $63(17.5)$ & $297(82.5)$ & $3.98(1.93,8.2)$ & $1.34(0.559,3.23)$ \\
\hline Do not accept & $9(5.1)$ & $169(94.9)$ & 1 & 1 \\
\hline \multicolumn{5}{|l|}{ Discussion making power } \\
\hline Low discussion making power & $45(25)$ & $135(75)$ & $4.09(2.44,6.86)$ & $1.56(0.807,3.012)$ \\
\hline $\begin{array}{l}\text { High discussion maker power } \\
\text { Wealth index }\end{array}$ & $27(7.5)$ & $331(92.5)$ & 1 & 1 \\
\hline Low & $11(6.1)$ & $168(93.9)$ & $0.279(0.14,0.57)$ & $0.935(0.355,2.46)$ \\
\hline Medium & $27(15)$ & $153(85)$ & $0.753(0.432,1.3)$ & $1.345(0.631,2.866)$ \\
\hline High & 34 & 145 & 1 & 1 \\
\hline
\end{tabular}

$*=\mathrm{p}<0.05 ; * *=\mathrm{p}<0.01 ; \mathrm{CI}=$ confidence interval; $\mathrm{COR}=$ crude odds ratio; $\mathrm{AOR}=$ adjusted odds ratio.

Women whose partner drinks alcohol daily were 3.3 times more likely to experience IPPV compared to those women whose intimate partner did not consume alcohol. This is supported by the findings in Uganda [29], in Jeddah, Saudi Arabia [30], in a Megacity of South Asia [23], findings in Ethiopia, such as in Gonder [31], Shirendasilase [16], and Shimelba [25]. This might be because alcohol consumption can affect consumers' cognitive functions and users to behave aggressively in their relationship and this may increase the occurrence of violence.

Pregnant women whose partner have other sexual partners were 3.77 times higher compared to women who reported that their partners did not have other sexual partners. This is consistent with the findings in Nigeria [32], and Uganda [29]. The possible reason might be due to the culture and religious value of the societies in which the social value permits and accepts only one to one relationships and polygamy is not accepted and considered as illegal in the community.

\section{Limitation}

There might be under-reporting due to the sensitive nature of IPV and cultural barriers.

\section{Conclusion}

The proportion of IPPV during the current pregnancy was $13.4 \%$. Being a rural dweller, being unmarried, spouses having multiple sexual partners, no interest in the current pregnancy by an intimate partner, and having alcohol drinker partners were found to be significant factors associated with IPPV during their current pregnancy. A significant number of pregnant women experienced physical violence by their intimate partner during recent pregnancy. Addressing male partner involvement to build positive relations between partners and sexual- reproductive health issues through intervention measures are very important to minimize victims of violence during pregnancy.

\section{Abbreviations}

ANC: Antenatal Care
IPPV: Intimate Partner Physical Violence

IPV: Intimate Partner Violence

SPSS: Statistical Package for Social Sciences

WHO: World Health Organization

\section{Data Availability}

The datasets used and/or analyzed during the current study are available from the principal author upon reasonable request.

\section{Ethical Approval}

Before the data collection process, ethical clearance was secured from the Haramaya University Institutional Health Research Ethics Review Committee. An official letter was written from Haramaya University to Ofla District Health Office and each public health facility.

\section{Consent}

Informed, voluntary, written and signed consent was obtained from each participant after explaining the purpose and benefits of the study. Respondents were informed that participating in this study is up to the willingness of them.

\section{Authors' Contributions}

GA was involved in the conception and design of the study, acquisition of data, analysis of data, interpretation of data, revising the paper, and writing of the manuscript. LO involved in advising during proposal development, data analysis, and revising the paper. TT revised the paper and reviewed a draft of the manuscript. All authors read and approved the final manuscript.

\section{Funding}

All costs of data collection and analysis were covered by the authors. 


\section{Conflicts of Interest}

The authors declare that they have no competing interests.

\section{Acknowledgements}

We want to express our heartfelt thanks to Haramaya University College of Health and Medical Sciences. We would like to thanks for Ofla/Korem District Health Office, for their cooperation and support, data collectors and supervisors for their responsible data collection and support. Authors would also greatly appreciate the study participants for their willingness to share their ideas and painful violence experiences.

\section{References}

[1] WHO. Global and regional estimates of violence against women: Prevalence and health effects of intimate partner violence and non-partner sexual violence. Geneva, Switzerland: Reproductive Health and Research. http://apps.who.int/iris/bitstream/10665/85240/1/9789241548 595_eng.2013.

[2] Devries K, Mak J, García MC, Petzold M, Child J, Falder G. The global prevalence of intimate partner violence against women: http://dx.doi.org/10.1126/science.1240937 Global health. 2013; 340 (6140): 1527-8.

[3] Helweg- Larsen K. Violence against women in Europe: magnitude and the mental health consequences described by different data sources. Violence against women and mental health. 2013; 178: 54-64.

[4] Bikines LT, Mash1 R, Joyner K. Prevalence of intimate partner violence and associated factors amongst women attending antenatal care at Outapi clinic, Namibia. African Journal of Primary Health Care \& Family Medicine 2017; 9: $1-6$.

[5] WHO. United Nations Development Programme; United Nations Office on Drugs and Crime. Global status report on violence prevention http://www.who.int/violence_injury_prevention/violence/statu S_report/2014/en/.2014.

[6] U N. Violence against women in South Asia. Violence against women and mental health. 2013; 178: 38-53.

[7] Division UNS. The world's women 2010. trends and statistics of 2010

[8] García-Moreno C, Jansen H, Ellsberg M, L. Heise, Watts CH. WHO multi-country study on women's health and domestic violence against women: initial results on prevalence, health outcomes and women's responses. 2005.

[9] KM D, S K, H J, H S, L B, C G-M. Intimate partner violence during pregnancy: prevalence data from 19 countries. Reproductive Health Matters. 2010; 18 (36): 1-13.

[10] S. Shamu, Abrahams N, Zarowsky C, Shefer T, Temmerman M. Intimate partner violence during pregnancy in Zimbabwe: a cross-sectional study of prevalence, predictors, and associations with HIV, Doi; 10.1111/tmi.12078. Tropical Medicine and International Health. 2013; 18: 696-711.
[11] EDHS. Central Statistical Agency; Ethiopian Demographic Health Survey Addis Ababa, Ethiopia and Rockville, Maryland, USA: CSA and ICF. 2016.

[12] CSA. (Ethiopia) and ORC Macro. Ethiopian demographic health survey Maryland: Ethiopia and Calverton 2012.

[13] WHO. Understanding and addressing violence against women. Intimate partner violence. Geneva, Switzerland.; 2012.

[14] Zemenu YK, Alemu WM. Physical violence and associated factors during pregnancy in Yirgalem town, South Ethiopia. Curr Pediatr Res. 2016; 20.

[15] SS J. Social determinants of married women's attitudinal acceptance of intimate partner violence. J Interpers Violence. 2015.

[16] Gebrezgi BH, Badi MB, Cherkose EA, Weldehaweria NB. "Factors associated with intimate partner physical violence among women attending antenatal care in Shire Endaselassie town, Tigray, northern Ethiopia: a cross-sectional study. Reproductive Health. 2017; 14 (1): 76.

[17] Ofla District health office annual report. 2018.

[18] WHO. Violence Against Women: intimate partner and sexual Violence Against Women Fact Sheet. http://www.who.int/mediacentre/factsheets/fs239/en/. 2016.

[19] Alam MS, Tareque MI, Peet ED, Rahman MM, Mahmud T. Female Participation in Household Decision Making and the Justification of Wife Beating in Bangladesh Journal of Interpersonal Violence. 2018: 1-20.

[20] Trott CD, Harman JJ, Kaufman MR. Women's Attitudes Toward Intimate Partner Violence in Ethiopia: The Role of Social Norms in the Interview Context. Violence Against Women. 2017; 23 (8): 1016-36.

[21] Abdollahi F, Abhari FR, Delavar MA, Charati JY. Physical violence against pregnant women by an intimate partner, and adverse pregnancy outcomes in Mazandaran Province, Iran. $J$ Family Community Med. 2015; 22 (1): 13-8.

[22] Tenaw Y, Tesfaye G, gudina E, habtamu M. Magnitude of Domestic Violence and Associated Factors among Pregnant Women in Hulet Ejju Enessie District, Northwest Ethiopia. Hindawi Publishing Corporation. 2014; 2014: 8.

[23] Ali N, Ali F, Khuwaja A, Nanji K. Factors associated with intimate partner violence Against women in a Megacity of South-Asia: multi-center cross-sectional study; 20, 297-303, DOI: 10.12809/hkmj13407. Hong Kong Med J. 2014; 20: 297.

[24] Malan M, Spedding MF, Sorsdahl K. The prevalence and predictors of intimate partner violence among pregnant women attending a midwife and obstetrics unit in the Western Cape. Global Mental Health 2018; 5 (18): 1-13.

[25] Feseha G, G/mariam A, Gerbaba M. Intimate partner physical violence among women in Shimelba refugee camp, northern Ethiopia. BMC Public Health. 2012; 12 (125).

[26] Rurangirwa AA, Mogren I, Ntaganira J, Krantz G. Intimate partner violence among pregnant women in Rwanda, its associated risk factors, and relationship to ANC services attendance. Biomedical Journal. 2017.

[27] Berhanu BB, Berihun AD, Bewket TT, Abarham ZZ. Domestic Violence among Pregnant Mothers in Northwest Ethiopia: Prevalence and Associated Factors. Advances in Public Health. 2017; 2017: 6. 
[28] B. O. Ahinkorah, K. S. Dickson, and A.-A. Seidu, "Women's decision-making capacity and intimate partner violence among women in sub-Saharan Africa," BMC, vol. 5, pp. 1-10, 2018.

[29] Namugamba A, Mangwi R. "Prevalence and factors associated with domestic violence during pregnancy in Arua district, Uganda," Iproceedings. 2015; 4: 1 (2018).

[30] Fageeh WMK. Factors associated with domestic violence: a cross sectional survey among women in Jeddah, Saudi Arabia. BMJ open 2014; 4: e004242. DOI: 10.1136/ bmj open-2013004242 .
[31] Fekadu E, Yigzaw G, Gelaye KA, Ayele TA, Minwuye T, Geneta $T$, et al. Prevalence of domestic violence and associated factors among pregnant women attending antenatal care service at University of Gondar Referral Hospital, Northwest Ethiopia. Biomed central Women's health. 2018; 18: 1-8.

[32] Ashimi AO, Amole TG. Prevalence and predictors for domestic violence among pregnant women in a rural community Northwest, Nigeria. PubMed. 2015; 56-2. 